\title{
Usefulness of digital velcro crackles detection in identification of interstitial lung disease in patients with connective tissue diseases
}

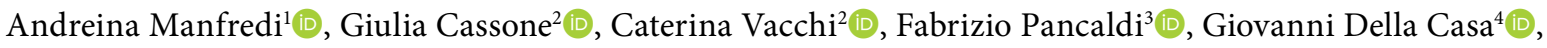

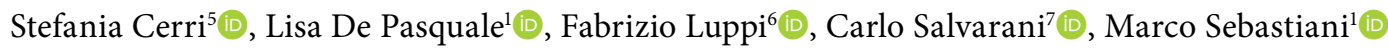 \\ ${ }^{1}$ Rheumatology Unit, University of Modena and Reggio Emilia, Modena, Italy \\ ${ }^{2}$ Department of Sciences and Methods For Engineering, University of Modena and Reggio Emilia, Modena, Italy \\ ${ }^{3}$ Radiology Unit, University of Modena and Reggio Emilia, Modena, Italy \\ ${ }^{4}$ Respiratory Unit, University of Modena and Reggio Emilia, Modena, Italy \\ ${ }^{5}$ Respiratory Unit, University of Milan-bicocca, San Gerardo Hospital, Monza, Italy \\ ${ }^{6}$ Rheumatology Unit, Irccs Arcispedale Santa Maria Nuova, Azienda Unità Sanitaria Locale-irccs Di Reggio Emilia, Reggio Emilia, Italy \\ ${ }^{7} \mathrm{PhD}$ Program In Clinical and Experimental Medicine, University of Modena and Reggio Emilia, Modena, Italy
}

\begin{abstract}
Objectives: This study aims to evaluate the diagnostic accuracy of the VECTOR software in patients with connective tissue diseases (CTDs), compared with the reference standard of high-resolution computed tomography (HRCT).

Patients and methods: The study included 98 consecutive patients of CTD (24 males, 74 females; median age: 66 years; range, 24 to 85 years) with a recent HRCT. Patients were evaluated in a blindly manner by VECTOR and the results obtained by the algorithm were compared with the presence of interstitial lung disease (ILD) according to HRCT.

Results: Interstitial lung disease was detected in $42.8 \%$ of subjects. VECTOR correctly classified $81 / 98$ patients, with a diagnostic accuracy of $82.6 \%$; sensitivity and specificity were $88.1 \%$ and $78.6 \%$, respectively. Only $5 / 42$ patients with ILD were not correctly classified by VECTOR, while false positive cases were $21.4 \%$. No significant differences were observed according to the radiologic pattern of ILD.

Conclusion: VECTOR showed high sensitivity, specificity and diagnostic accuracy, allowing selecting patients to be investigated with HRCT. The relatively high frequency rate of false positive results is acceptable if compared with the lack of effective screening methods for this complication of CTDs.

Keywords: Connective tissue diseases, diagnosis, interstitial lung disease, screening, VECTOR, velcro crackles.
\end{abstract}

Interstitial lung disease (ILD) represents one of the more frequent pulmonary manifestations in connective tissue diseases (CTDs) and it is characterized by severe implications in morbility and overall prognosis. ${ }^{1-3}$ To date, high-resolution computed tomography (HRCT) represents the gold standard for the diagnosis and the characterization of ILD ${ }^{4}$ moreover, an increasing role for radiology has been observed in the last years; it allows to classify the different possible patterns with high specificity ${ }^{5}$ and to quantify the evolution over the time of the lung disease. ${ }^{6}$

Received: December 24, 2019 Accepted: February 14, 2020 Published online: June 25, 2020

Correspondence: Marco Sebastiani, MD. PhD Program In Clinical and Experimental Medicine, University of Modena and Reggio Emilia, 41124 Modena, Italy. Tel: +390594222595 e-mail: marco.sebastiani@unimore.it

\section{Citation:}

Manfredi A, Cassone G, Vacchi C, Pancaldi F, Casa GD, Cerri S, et al. Usefulness of digital velcro crackles detection in identification of interstitial lung disease in patients with connective tissue diseases. Arch Rheumatol 2021;36(1):19-25.

This is an open access article under the terms of the Creative Commons Attribution-NonCommercial License. which permits use. distribution and reproduction in any medium. provided the original work is properly cited and is not used for commercial purposes (http://creativecommons.org/licenses/by-nc/4.0/). 
Despite the potentially deep impact of lung involvement on clinical course and prognosis, a routine assessment of ILD is not properly defined for all CTDs. It is probably better defined in patients with systemic sclerosis (SSc), ${ }^{7}$ while it is not so common for other CTDs, such as primitive Sjögren's syndrome (SS) or systemic lupus erythematosus (SLE) that can also be complicated by ILD. ${ }^{1,8-10}$ Moreover, increasing data suggest a higher prevalence than expected of severe ILD, also in CTDs other than SSc; nevertheless, the absence of a routine screening for ILD can frequently delay the diagnosis..11,12 On the other side, the routine use of HRCT is not feasible for the high costs and radiation exposure and alternative screening methods are needed.

Clinical examination and lung auscultation are mandatory for detection of pulmonary disease, and velcro-type crackles have been considered typical of lung fibrosis and early detectable in the course of the disease..$^{13-16}$ Crackles, best detected during slow, deep breaths, are discontinuous, short explosive non-musical sounds predominating during inspiration and best heard over dependent lung regions. Fine crackles are softer, shorter in duration, and higher in pitch than coarse crackles, similar to the sound heard when gently separating the joined strip of velcro on the blood pressure cuff. Crackles are present early in the course of idiopathic pulmonary fibrosis (IPF), appearing first in the basal areas of the lung where the disease process initiates, with further progression to the upper zones. Although not specifically studied according to the stage of IPF, crackles may be present in virtually any patient with IPF according to current diagnostic criteria. ${ }^{13-17}$ For these reasons, they have been proposed as a simple and reliable screening for the early diagnosis of ILD. ${ }^{13-16}$

Recently, Sgalla et al. ${ }^{13}$ observed a close correlation between pulmonary fibrosis and "velcro-type" crackles; in their study reticulation, honeycombing, ground-glass opacities, and traction bronchiectasis were all independently associated with velcro-type crackles in the lung parenchyma.

More recently, we developed the software VECTOR (VElcro Crackles detecTOR) which is able to identify velcro crackles in pulmonary sounds recorded by an electronic stethoscope (ES) in rheumatoid arthritis (RA) patients. This previous study was performed on 137 RA patients and showed a diagnostic accuracy for VECTOR of $83.9 \%$ with a specificity and sensitivity of $76.9 \%$ and $93.2 \%$, respectively. ${ }^{14}$ However, the results obtained in RA cannot be translated in CTDs, because of the heterogeneity of clinical and radiological manifestations of lung involvement in these diseases. Therefore, in this study, we aimed to evaluate the diagnostic accuracy of the VECTOR software in patients with CTDs, compared with the reference standard of HRCT.

\section{PATIENTS AND METHODS}

This study was conducted at University Hospital of Modena between September 2018 and April 2019. The study included 98 consecutive patients of CTD (24 males, 74 females; median age: 66 years; range, 24 to 85 years). All patients satisfying the current classification criteria for a CTD, namely dermatomyositis (DM), SSc, primitive SS, antisynthetase syndrome, SLE, and undifferentiated connective tissue disease (UCTD) ${ }^{18-22}$ and those who underwent HRCT in the last 12 months before the study were included. Overlap syndromes and secondary forms of SS were excluded. The appearance of symptoms suggestive for lung disease (cough, dyspnea) in patients without a previous history of ILD or the presence of pleural effusion or pneumothorax at HRCT also entailed the exclusion by the study; when possible, a new HRCT was requested. The study protocol was approved by the University Hospital of Modena Ethics Committee. A written informed consent was obtained from each patient. The study was conducted in accordance with the principles of the Declaration of Helsinki.

All patients were evaluated in a blinded manner by means of VECTOR and the results (presence or absence of velcro crackles) were compared with HRCT (presence or absence of ILD).

According to our previous study, lung auscultation was performed bilaterally in four pulmonary fields (two at the basal fields and one at the medium and upper fields, respectively; Figure 1). Auscultations were realized in a silent environment, with a commercially available ES (Littmann 3200TM 3M, St. Paul, MN, USA). 


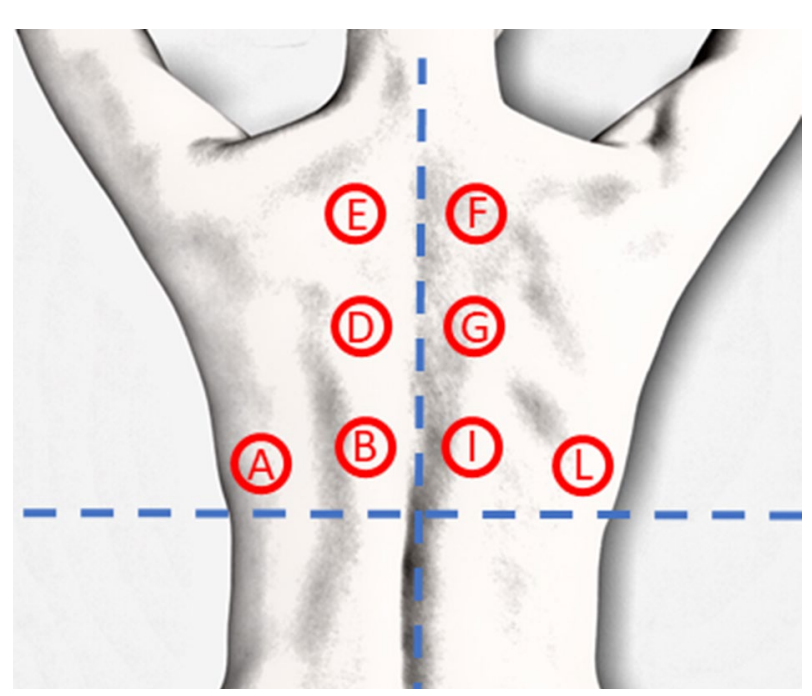

Figure 1. Lung auscultation. All patients were auscultated bilaterally in four pulmonary fields at the dorsal level: two at the basal field, one at the middle field and one at the upper field.

Lung auscultation. All patients were auscultated bilaterally in four pulmonary fields at the dorsal level: two at the basal field ( $\mathrm{A}$ and $\mathrm{B}$, I and $\mathrm{L}$ ), one at the middle field (D and $\mathrm{G}$ ) and one at the upper field (E and F).

Then, registrations were digitized, anonymized, saved as a Waveform Audio file, and analyzed by means of VECTOR. ${ }^{14}$

Similarly, all HRCT images were anonymized and transferred on Digital Imaging and Communications in Medicine format, coded, and evaluated for the assessment of ILD by an expert thoracic radiologist ${ }^{5}$ blinded to the clinical condition of the patients and the presence of velcro crackles.

All HRCTs were performed by means of different multidetector scanners with a slice thickness of less than $2 \mathrm{~mm}$, from the lung apices to below the costophrenic angles, reconstructed by using an edge-enhancing algorithm. The scan was performed in the supine position at full inspiration. All images were viewed at window setting optimized for assessment of lung parenchyma (width $1500 \mathrm{HU}$; level $-700 \mathrm{HU}$ ). High-resolution computed tomography images were evaluated by an expert chest radiologist who classified the radiologic ILD pattern according to American Thoracic Society (ATS)/ European Respiratory Society (ERS)/Japanese Respiratory Society (JRS)/Latin American Thoracic Association (ALAT) statement for the diagnosis of IPF. ${ }^{5}$ The pattern of interstitial pneumonia was classified as definite, possible, and inconsistent with usual interstitial pneumonia (UIP) (Table 1). If a pattern inconsistent with UIP was recorded, the radiologist further specified if it was compatible with an organizing pneumonia, nonspecific interstitial pneumonia, or lymphoid interstitial pneumonia (LIP). ${ }^{5}$ Moreover, in the absence of ILD, the presence of nodules, pleural effusion or other isolated manifestations of pulmonary disease such as consolidation was also recorded.

\section{Statistical analysis}

Data were reported as median and interquartile range and analyzed using STATA version 11 statistical software (StataCorp. LP, College Station, Texas, USA). chi-square test was used to analyze categorical variables, while differences between the medians were evaluated using the Mann-Whitney U test for unpaired samples. Finally, we calculated the diagnostic accuracy, sensitivity, and specificity of the VECTOR. $P$ values $<0.05$ were considered statistically significant.

\section{RESULTS}

Of the CTD patients enrolled, seven had DM, 32 had SSc, 29 had primitive SS, six had antisynthetase syndrome, eight had SLE, and 16 had UCTD. The indications for the HRCT were presence of velcro crackles at lung auscultation (23.4\%), dyspnea (22\%), a suggestive thorax X-ray (14.1\%), and cough (4\%). In the remaining patients, HRCT was requested for other reasons including infections, screening for tumor or other lung diseases, and monitoring of lung nodules. Clinical, demographic, and serological characteristics of the 98 patients investigated are described in Table 1.

An ILD was detected in 42 patients (42.8\%). VECTOR showed a diagnostic accuracy of $82.6 \%$, classifying in a correct manner $81 / 98$ patients; sensitivity and specificity were $88.1 \%$ and $78.6 \%$, respectively. Seventeen patients were not correctly classified by VECTOR: 5/42 among patients with ILD, and 12/56 among control group. Among the latter, the majority showed signs of airway disease (8/12) while two patients presented an acute inflammatory event. 
Table 1. Demographic and clinico-radiological features of connective tissue disease patients

\begin{tabular}{|c|c|c|c|c|c|c|c|c|c|c|c|c|c|}
\hline & \multicolumn{4}{|c|}{ Non-ILD } & \multicolumn{4}{|c|}{ ILD } & \multicolumn{4}{|c|}{ Total } & \multirow[b]{2}{*}{$p$} \\
\hline & $\mathrm{n}$ & $\%$ & Median & IQR & $\mathrm{n}$ & $\%$ & Median & IQR & $\mathrm{n}$ & $\%$ & Median & IQR & \\
\hline Number & & 57.2 & & & 42 & 42.8 & & & 98 & & & & \\
\hline \multicolumn{14}{|l|}{ Sex } \\
\hline Female & & 73.2 & & & 33 & 78.6 & & & 74 & 75.5 & & & 0.61 \\
\hline Median age (year) & & & 64 & 21 & & & 68.5 & 13 & & & 66 & 18 & 0.08 \\
\hline Disease duration (month) & & & 60 & 112 & & & 78.5 & 99 & & & 72 & 103 & 0.58 \\
\hline Smoking & & 76.8 & & & 32 & 76.2 & & & 75 & 76.5 & & & 0.96 \\
\hline Antinuclear antibodies & 53 & 94.6 & & & 39 & 92.8 & & & 92 & 92.8 & & & 0.91 \\
\hline ENA & 39 & 69.6 & & & 29 & 69.0 & & & 68 & 69.4 & & & 0.98 \\
\hline Anti-SSA & 25 & 44.6 & & & 19 & 45.2 & & & 44 & 44.9 & & & 0.89 \\
\hline Rheumatoid factor & 10 & 17.8 & & & 9 & 21.4 & & & 19 & 19.4 & & & 0.79 \\
\hline \multicolumn{14}{|l|}{ Pattern HRCT } \\
\hline UIP & - & - & & & 19 & 19.4 & & & 19 & 19.4 & & & \\
\hline $\mathrm{OP}$ & - & - & & & 6 & 6.1 & & & 6 & 6.1 & & & \\
\hline NSIP & - & - & & & 13 & 13.3 & & & 13 & 13.3 & & & \\
\hline LIP & - & - & & & 1 & 1 & & & 1 & 1 & & & \\
\hline Other & - & - & & & 3 & 3.1 & & & 3 & 3.1 & & & \\
\hline \multicolumn{14}{|l|}{ Rheumatic diseases } \\
\hline DM & 3 & 5.3 & & & 4 & 9.5 & & & 7 & 7.1 & & & \\
\hline SLE & 7 & 12.5 & & & 1 & 2.3 & & & 8 & 8.2 & & & \\
\hline ASSD & 2 & 3.6 & & & 4 & 9.5 & & & 6 & 6.1 & & & \\
\hline pSS & 21 & 37.5 & & & 8 & 19.0 & & & 29 & 29.6 & & & \\
\hline SSC & 10 & 17.8 & & & 22 & 52.4 & & & 32 & 32.6 & & & \\
\hline UCTD & 13 & 23.2 & & & 3 & - & & & 16 & 16.3 & & & \\
\hline
\end{tabular}

Table 2. Diagnostic accuracy according to high-resolution computed tomography pattern

\begin{tabular}{lccc}
\hline & $\mathrm{n}$ & $\%$ & Diagnostic accuracy (\%) \\
\hline Usual interstitial pneumonia & 19 & 19.4 & 89.5 \\
Nonspecific interstitial pneumonia & 13 & 13.3 & 92.3 \\
Organizing pneumonia & 6 & 6.1 & 100 \\
Lymphoid interstitial pneumonia & 1 & 1 & 100 \\
Other & 3 & 3.1 & 100 \\
Normal & 56 & 57.1 & 89.8 \\
\hline
\end{tabular}

Among false negative cases, three patients had very advanced lung disease and the result of VECTOR probably derived from the difficulty to breathe deeply properly. The other two false negative cases included a patient with LIP and a patient with the only evidence of ground-glass at HRCT (without clear signs of fibrosis). No significant differences were observed according to the radiologic pattern of ILD (Table 2).

\section{DISCUSSION}

Interstitial lung disease represents one of the most harmful clinical manifestations in the course of CTDs, associated to very poor quality of life and increased mortality. ${ }^{1-3}$

Although CTD-ILD represents an important field of research for both pulmonologists and rheumatologists, there is a lack of prospective data about prevalence, follow-up, and efficacy 
of the treatment. For this reason, we may speculate that the data concerning prognosis can underestimate the real impact of the disease.

According to the available literature data, ILD can occur in every stage of the disease, sometimes preceding the diagnosis of the rheumatic disease and sometimes in long-standing CTDs. ${ }^{11,23}$ At the moment, a screening for ILD secondary to rheumatic disease is not feasible mainly because of the X-ray exposure and high cost of HRCT; moreover, any method other than HRCT shows a relatively low diagnostic accuracy, resulting in mis- or delayed diagnosis., ${ }^{44-26}$

The present study confirmed the high diagnostic accuracy, sensitivity, and specificity of VECTOR in the identification of ILD in CTD patients. Our previous study conducted in patients with RA showed a very good diagnostic accuracy (83.9\%) for VECTOR, and a specificity and sensitivity of $76.9 \%$ and 93.2\%, respectively, considering HRCT as the reference method for diagnosis of ILD. ${ }^{14,15}$ Despite the higher heterogeneity of ILD in CTDs rather than in RA, the specificity of VECTOR remained very high. At the same time, the sensitivity of the software was also preserved and it allowed us to identify virtually all patients with ILD. In fact, only five patients were not detected by VECTOR, and, among them, three had very advanced pulmonary disease while their clinical status allowed a correct identification.

VECTOR showed a relatively high frequency rate of false positive results; nevertheless, we can consider this acceptable compared with the lack of effective screening methods for this severe complication of CTDs. As reported by Cottin and Cordier, although crackles are not specific for IPF, they must prompt a thorough diagnostic process. They may occasionally be heard in healthy individuals ${ }^{27,28}$ or in patients with congestive heart failure. ${ }^{29}$ Moreover, crackles may also be heard occasionally in patients with chronic obstructive pulmonary disease or bronchiectasis, probably due to greater traction forces being exerted on the small airways. ${ }^{30}$ In these cases, patients' clinical history and condition may assist in discriminating those with fibrosing lung involvement. The introduction of an easy-touse tool helpful in the screening of patients to undergo HRCT evaluation can significantly support early diagnosis improving clinical and therapeutic management of CTD-ILD patients while reducing the cost of inappropriate radiological evaluations.

The retrospective design represents the main limitation of the study; however, it was decided to avoid further exposure of patients to $\mathrm{X}$-rays and to use the recent radiological data available. ${ }^{14}$ Nevertheless, prospective studies are required to compare the predictive value of VECTOR with other possible screening methods, such as lung ultrasonography ${ }^{31}$ or pulmonary function tests. ${ }^{32}$

In conclusion, we believe that VECTOR can efficiently support the clinical practice of rheumatologists involved in the care of CTD patients. This device can be helpful in the enrolment of CTD-ILD patients for prospective studies to clarify certain epidemiological, clinical, and prognostic needs in these diseases.

\section{Declaration of conflicting interests}

The authors declared no conflicts of interest with respect to the authorship and/or publication of this article.

\section{Funding}

The authors received no financial support for the research and/or authorship of this article.

\section{REFERENCES}

1. Antin-Ozerkis D, Hinchcliff M. Connective Tissue Disease-Associated Interstitial Lung Disease: Evaluation and Management. Clin Chest Med 2019;40:617-36.

2. Manfredi A, Sebastiani M, Cerri S, Vacchi C, Tonelli R, Della Casa G, et al. Acute exacerbation of interstitial lung diseases secondary to systemic rheumatic diseases: a prospective study and review of the literature. J Thorac Dis 2019;11:1621-8.

3. Ratwani AP, Ahmad KI, Barnett SD, Nathan SD, Brown AW. Connective tissue disease-associated interstitial lung disease and outcomes after hospitalization: A cohort study. Respir Med 2019;154:1-5.

4. Paschalaki KE, Jacob J, Wells AU. Monitoring of Lung Involvement in Rheumatologic Disease. Respiration 2016;91:89-98. 
5. Travis WD, Costabel U, Hansell DM, King TE Jr, Lynch DA, Nicholson AG, et al. An official American Thoracic Society/European Respiratory Society statement: Update of the international multidisciplinary classification of the idiopathic interstitial pneumonias. Am J Respir Crit Care Med 2013;188:733-48.

6. Takei R, Arita M, Kumagai S, Ito Y, Tokioka F, Koyama T, et al. Radiographic fibrosis score predicts survival in systemic sclerosis-associated interstitial lung disease. Respirology 2018;23:385-91.

7. Gasperini ML, Gigante A, Iacolare A, Pellicano $\mathrm{C}$, Lucci $\mathrm{S}$, Rosato $\mathrm{E}$. The predictive role of lung ultrasound in progression of scleroderma interstitial lung disease. Clin Rheumatol 2020;39:119-23.

8. Wells A, Devaraj A, Renzoni EA, Denton CP. Multidisciplinary Evaluation in Patients with Lung Disease Associated with Connective Tissue Disease. Semin Respir Crit Care Med 2019;40:184-93.

9. Dong X, Zhou J, Guo X, Li Y, Xu Y, Fu Q, et al. A retrospective analysis of distinguishing features of chest HRCT and clinical manifestation in primary Sjögren's syndrome-related interstitial lung disease in a Chinese population. Clin Rheumatol 2018;37:2981-8.

10. Cobo-Ibáñez T, López-Longo FJ, Joven B, Carreira PE, Muñoz-Fernández S, Maldonado-Romero V, et al. Long-term pulmonary outcomes and mortality in idiopathic inflammatory myopathies associated with interstitial lung disease. Clin Rheumatol 2019;38:803-15.

11. Wells A, Denton CP. Pulmonary Complications of Connective Tissue Disease. Semin Respir Crit Care Med 2019;40:145-6.

12. Ciancio N, Pavone M, Torrisi SE, Vancheri A, Sambataro D, Palmucci S, et al. Contribution of pulmonary function tests (PFTs) to the diagnosis and follow up of connective tissue diseases. Multidiscip Respir Med 2019;14:7.

13. Sgalla G, Walsh SLF, Sverzellati N, Fletcher S, Cerri $\mathrm{S}$, Dimitrov B, et al. "Velcro-type" crackles predict specific radiologic features of fibrotic interstitial lung disease. BMC Pulm Med 2018;18:103.

14. Manfredi A, Cassone G, Cerri S, Venerito V, Fedele $\mathrm{AL}$, Trevisani M, et al. Diagnostic accuracy of a velcro sound detector (VECTOR) for interstitial lung disease in rheumatoid arthritis patients: the InSPIRAtE validation study (INterStitial pneumonia in rheumatoid ArThritis with an electronic device). BMC Pulm Med 2019;19:111.

15. Dawson JK, Fewins HE, Desmond J, Lynch MP, Graham DR. Predictors of progression of HRCT diagnosed fibrosing alveolitis in patients with rheumatoid arthritis. Ann Rheum Dis 2002;61:51721.

16. Cottin V, Cordier JF. Velcro crackles: the key for early diagnosis of idiopathic pulmonary fibrosis? Eur Respir J 2012;40:519-21.
17. Raghu G, Collard HR, Egan JJ, Martinez FJ, Behr J, Brown KK, et al. An official ATS/ERS/ JRS/ALAT statement: idiopathic pulmonary fibrosis: evidence-based guidelines for diagnosis and management. Am J Respir Crit Care Med 2011;183:788-824.

18. van den Hoogen F, Khanna D, Fransen J, Johnson SR, Baron M, Tyndall A, et al. 2013 classification criteria for systemic sclerosis: an American college of rheumatology/European league against rheumatism collaborative initiative. Ann Rheum Dis 2013;72:1747-55.

19. Shiboski CH, Shiboski SC, Seror R, Criswell LA, Labetoulle M, Lietman TM, et al. 2016 American College of Rheumatology/European League Against Rheumatism classification criteria for primary Sjögren's syndrome: A consensus and data-driven methodology involving three international patient cohorts. Ann Rheum Dis 2017;76:9-16.

20. Bohan A, Peter JB. Polymyositis and dermatomyositis (first of two parts). N Engl J Med 1975;292:344-7.

21. Aringer M, Costenbader K, Daikh D, Brinks R, Mosca M, Ramsey-Goldman R, et al. 2019 European League Against Rheumatism/American College of Rheumatology Classification Criteria for Systemic Lupus Erythematosus. Arthritis Rheumatol 2019;71:1400-12.

22. Mosca M, Neri R, Bombardieri S. Undifferentiated connective tissue diseases (UCTD): a review of the literature and a proposal for preliminary classification criteria. Clin Exp Rheumatol 1999;17:615-20.

23. Long K, Danoff SK. Interstitial Lung Disease in Polymyositis and Dermatomyositis. Clin Chest Med 2019;40:561-72.

24. Manfredi A, Sebastiani M, Cerri S, Cassone G, Bellini P, Della Casa G, et al. Erratum to: Prevalence and characterization of non-sicca onset primary Sjögren syndrome with interstitial lung involvement. Clin Rheumatol 2017;36:1931.

25. Bongartz T, Nannini C, Medina-Velasquez YF, Achenbach SJ, Crowson CS, Ryu JH, et al. Incidence and mortality of interstitial lung disease in rheumatoid arthritis: a population-based study. Arthritis Rheum 2010;62:1583-91.

26. Iqbal K, Kelly C. Treatment of rheumatoid arthritisassociated interstitial lung disease: a perspective review. Ther Adv Musculoskelet Dis 2015;7:247-67.

27. Thacker RE, Kraman SS. The prevalence of auscultatory crackles in subjects without lung disease. Chest 1982;81:672-4.

28. Workum P, Holford SK, Delbono EA, Murphy RL. The prevalence and character of crackles (rales) in young women without significant lung disease. Am Rev Respir Dis 1982;126:921-3.

29. Kataoka H, Matsuno O. Age-related pulmonary crackles (rales) in asymptomatic cardiovascular patients. Ann Fam Med 2008;6:239-45. 
30. Piirilä P, Sovijärvi AR, Kaisla T, Rajala HM, Katila T. Crackles in patients with fibrosing alveolitis, bronchiectasis, COPD, and heart failure. Chest 1991;99:1076-83.

31. Moazedi-Fuerst FC, Kielhauser S, Brickmann K, Tripolt N, Meilinger M, Lufti A, et al. Sonographic assessment of interstitial lung disease in patients with rheumatoid arthritis, systemic sclerosis and systemic lupus erythematosus. Clin Exp Rheumatol 2015;33:S87-91.

32. Dawson JK, Fewins HE, Desmond J, Lynch MP, Graham DR. Fibrosing alveolitis in patients with rheumatoid arthritis as assessed by high resolution computed tomography, chest radiography, and pulmonary function tests. Thorax 2001;56:622-7. 